\title{
Development and Impacts of the Sierra Leone-China Laboratory for Parasitic Diseases Testing and Surveillance
}

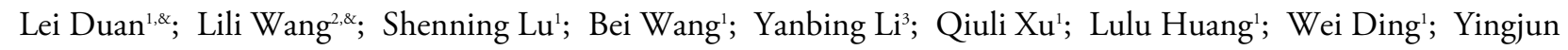
Qian'; Hongmei Li'; Xuejiao Ma'; Duoquan Wang'; Yayi Guan'; Xiaochun Wang²; Ning Xiaoo ${ }^{1, *}$; Xiao-nong Zhou'

\section{BACKGROUND}

The Republic of Sierra Leone is located on the west coast of Africa, bordering Guinea and Liberia, with a population of 7.8 million in 2019 (1). It has a tropical monsoon climate, with high temperatures and plentiful precipitation. As one of the least developed countries in the world (2), Sierra Leone has long struggled with poor health outcomes with a life expectancy at birth of 59 for male and 61 for female, and high maternal and neonatal mortality, threatened by infectious diseases such as malaria, tuberculosis, typhoid fever, cholera, and Lassa fever (3).

Since March 2014, the 3 West African countries of Sierra Leone, Guinea, and Liberia have been hit by the worst outbreak of Ebola virus disease (EVD) in history. A total of 28,610 cases and 11,308 deaths were reported (4). As requested, the Chinese government has rapidly sent 1,200 medical staff to these countries to fight against Ebola (5). In a bid to conduct testing for Ebola and other viral hemorrhagic fevers (VHF), China CDC established the Sierra Leone-China Friendship Fixed Biological Safety Laboratory Level 3 (BSL-3 Laboratory) in Sierra Leone, which was officially put in operation in March 2015. It now serves as the national reference laboratory for VHF in Sierra Leone and the national training center for virus testing and biosafety. Since then, China and Sierra Leone have implemented 2 technical cooperation projects (Phase 1 and Phase 2) based on the BSL-3 Laboratory to provide technical support to Sierra Leone in terms of VHF testing capacity and surveillance capacity of key infectious diseases.

Sierra Leone also suffers from a heavy burden of parasitic diseases, including malaria, schistosomiasis, lymphatic filariasis, onchocerciasis, soil-transmitted helminth diseases, and African trypanosomiasis (6). The Global Burden of Disease Study 2019 showed that the malaria burden in Sierra Leone in 2019 was $824,000(355,000-1,400,000)$ disability-adjusted life years (DALYs) per 100,000 individuals, and the onchocerciasis burden was $23,000(9,750-40,000)$ DALYs per 100,000 individuals (7). According to the World Malaria Report 2020, Sierra Leone reported 2,615,850 malaria cases in 2019 with an average incidence rate of $33.5 \%(2,615,850 / 7,813,207)$, and 6,824 malaria deaths with a case fatality rate of $0.3 \%$ $(6,824 / 2,615,850)$. It is estimated that 2.24 million outpatient visits each year were due to malaria, of which about 1 million were children under 5 years old (8). Parasitic diseases, especially malaria, have posed great threats to health and socioeconomic development in Sierra Leone.

At present, Sierra Leone faces huge challenges in the prevention and control of parasitic diseases. First, many weaknesses exist in its public health system, including laboratory testing capacity, implementation of prevention and control measures, prevention and control personnel, and data quality. Second, prevention and control efforts fall short of strong fundamentals with few national epidemiological data and incomplete vector monitoring data. Third, the parasitic disease control program is unsustainable and needs more investment as it is largely dependent on international aid projects.

To understand the disease spectrum and infection status of parasitic diseases in Sierra Leone, the Sierra Leone-China BSL-3 laboratory for parasitic disease testing and surveillance (parasitic disease laboratory) was jointly established by China CDC and Ministry of Health and Sanitation of the Republic of Sierra Leone. This paper reviews the establishment, development, and impacts of the BSL-3 laboratory.

\section{ESTABLISHMENT PROCESS AND WORK CONTENT}

When the BSL-3 Laboratory Technical Cooperation Project Phase 1 (July 2015-June 2017) was underway, China CDC had realized the importance of malaria infection and planned to build the laboratory's testing capacity for malaria. To promote the convenience and 
efficiency of testing, a special laboratory for parasitic diseases was set up in the Sierra Leone-China Friendship Hospital in July 2017 during the implementation of BSL-3 Laboratory Technical Cooperation Project Phase 2, in which febrile patients were tested for malaria.

The parasitic disease laboratory is designed in accordance with the standards of a Biosafety Level 2 Laboratory. With one year's efforts, the laboratory has established basic conditions for malaria detection and mosquito vector identification. The development history of the laboratory was shown in Figure 1.

\section{ACHIEVEMENTS AND EXPERIENCES}

Supported by the BSL-3 Laboratory Technical Cooperation Project Phase 2, 10 general hospitals were selected as sentinel hospitals in 3 regions of Sierra Leone (later expanded to 13 sentinel hospitals in 6 regions).

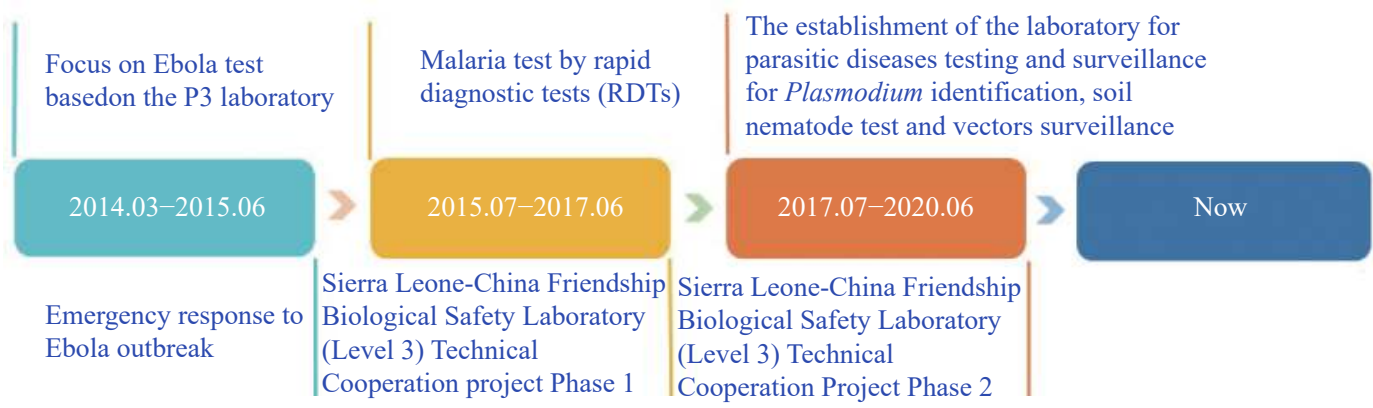

FIGURE 1. Milestones of Sierra Leone-China laboratory for parasitic diseases testing and surveillance.

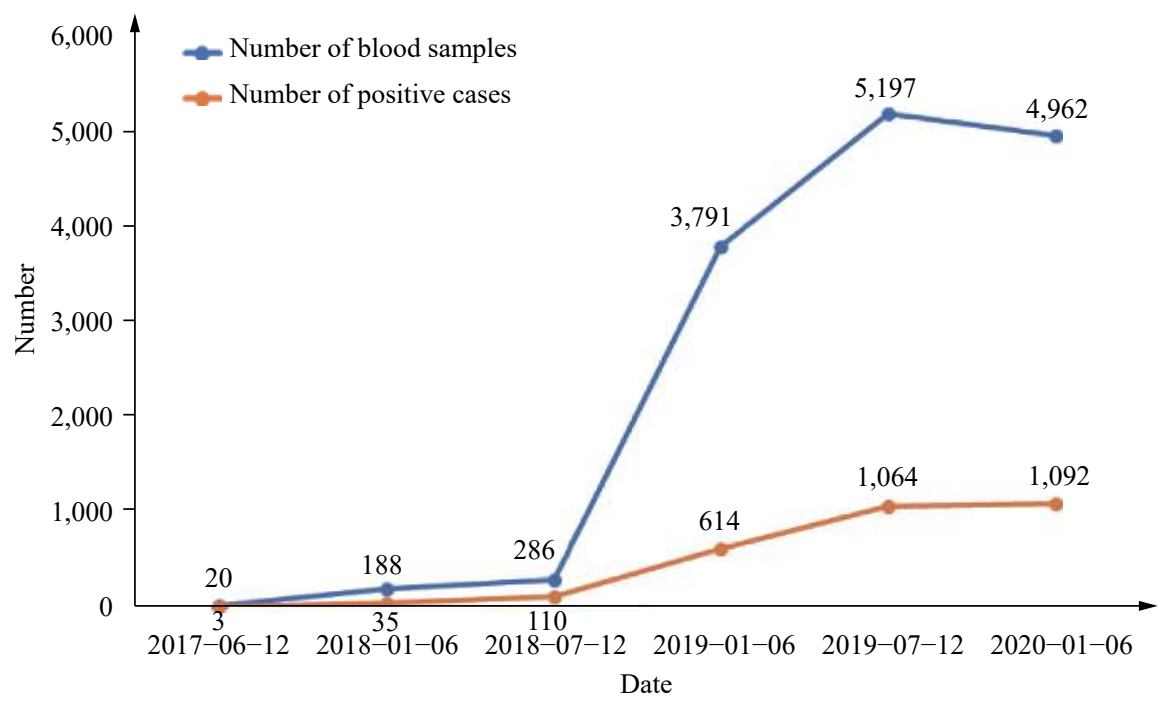

FIGURE 2. Number of blood samples and malaria-positive cases tested by the Sierra Leone-China laboratory for parasitic disease testing and surveillance from 2017 to 2020.
During June 2017 to June 2020, relying on the BSL-3 Laboratory, the parasitic disease laboratory has detected 14,444 blood samples of febrile patients from sentinel hospitals by rapid diagnostic tests (RDTs) for malaria, of which 2,918 were positive with a positivity rate of $20.2 \%(2,918 / 14,444)$ (Figure 2).

A total of 413 RDT-test-positive blood samples were applied for species identification by microscopy, in which the Plasmodium. falciparum infection accounted for 56.2\% (232/413), other Plasmodium excluded $P$. falciparum (PAN) infection accounted for $4.3 \%$ (18/413), mixed infection with $P$. falciparum and other Plasmodium accounted for $39.5 \%$ (163/413). The results demonstrated that $P$. falciparum was a dominant pathogen of malaria in Sierra Leone.

The Kato-Katz technique was deployed to test the stool samples for soil-transmitted helminth infections, with the equipment and expertise of the parasitic disease laboratory in collaboration with the Chinese medical team. The results indicated that the ascaris and

ishment of the laboratory for

\section{$2017.07-2020.06$}

(Level 3) Technical

Cooperation Project Phase 2 
hookworm eggs positive rate was $40.0 \%$ (5/20). At the same time, the laboratory carried out a 3-year plan for monitoring Anopheles mosquitoes at 10 field sites in the western urban areas and suburban areas of Sierra Leone's capital. According to morphological identification, out of 22,947 mosquito samples, Anopheles (An. gambiae s.s., An. funestus, and An. melas) accounted for $15.3 \%$ (Figure 3 ).

The parasitic disease laboratory serves as a reference laboratory for parasite identification in Sierra Leone. It provides timely technical support for patient treatment. The laboratory once carried out a molecular analysis of a blood sample from a Chinese patient with severe artesunate hemolysis who was later confirmed with $P$. falciparum infection. In 2017, when a mudslide hit Freetown, the capital city of Sierra Leone, the experts from the laboratory played a crucial role in diseases and vectors surveillance, evaluation, and disinfection. Moreover, the laboratory also works on mosquito vector monitoring in many high-risk communities of Sierra Leone and Chinese-funded enterprises.

The parasitic disease laboratory is dedicated to the detection and monitoring of parasites and tropical diseases, which further enhances work efficiency and resource allocation of the BSL-3 laboratory. In Sierra Leone, a country with a high burden of parasitic diseases accompanied by VHF such as Ebola and Lassa fever, establishing a specialized laboratory for parasitic disease detection based on the BSL-3 laboratory model is crucial to strengthening the public health systems.
From the perspective of biosafety, the nucleic acid extraction of samples from the Lassa fever epidemic area could be completed in a P3 laboratory and then transferred to the parasitic disease laboratory for testing. Samples of suspected cases from other areas can be directly tested in the parasitic disease laboratory. In terms of technology and talents, the technical staff were recruited from medical colleges and universities in Sierra Leone or recommended by the Ministry of Health and Sanitation of Sierra Leone. These staff were first dispatched to parasitic disease laboratories at a lower biosafety level, and later some high-achieving individuals ones were selected to work in the BSL-3 laboratory. This upward movement of personnel fits the present status of their public health capacity in Sierra Leone.

The laboratory has laid a solid foundation for bilateral and multilateral cooperation projects in public health, as it is a platform for Sierra Leone and other international organizations to learn more about China's products and experience in the prevention and control of parasitic diseases. Based on previous achievements, the Bill and Melinda Gates Foundation has decided to support China CDC to implement molecular epidemiological research on malaria in Sierra Leone.

\section{CHALLENGES AND PERSPECTIVE}

In 2005, the World Health Organization (WHO) recommended artemisinin-based combination

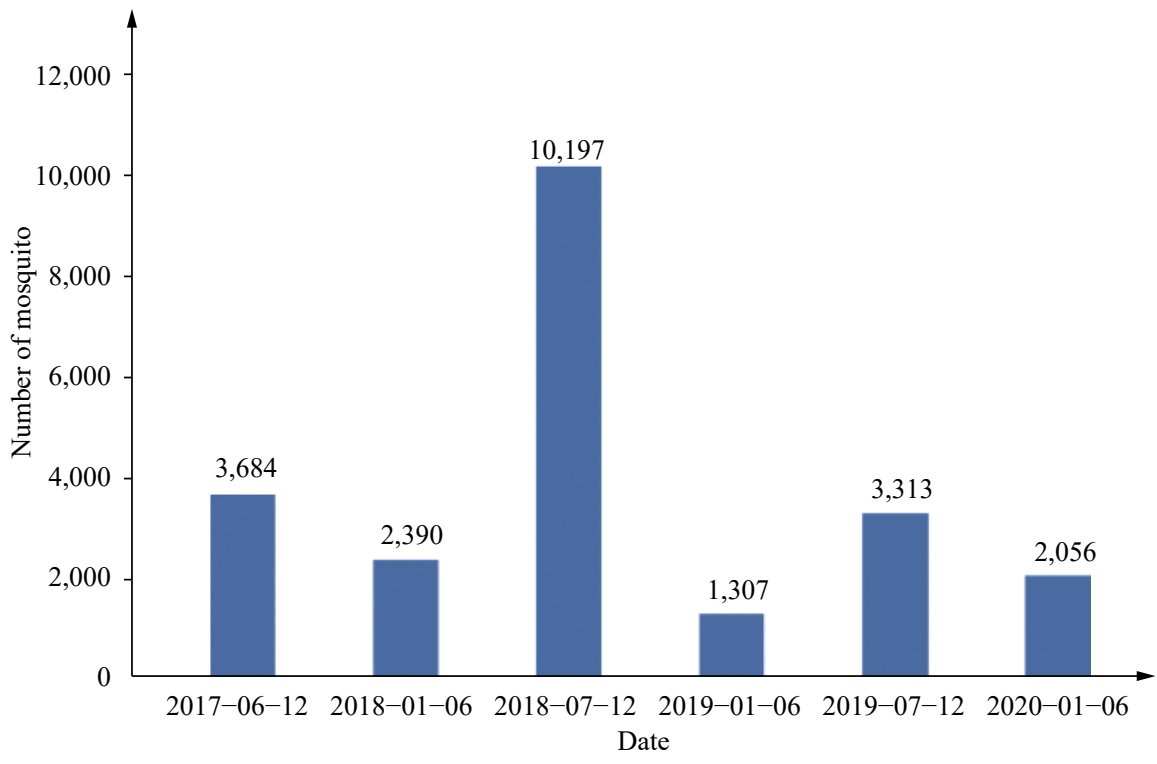

FIGURE 3. Identification number of mosquito vectors by the Sierra Leone-China laboratory for parasitic disease testing and surveillance from 2017 to 2020 . 
therapies as first-line treatments for falciparum malaria in endemic areas (9). However, artemisinin resistance has been reported in Rwanda and other countries (10). If resistant strains continue to spread, the lives of tens of thousands of African patients could be at risk. Hence, it is an urgent task to monitor the Plasmodiumresistance to artemisinin, which remains a huge challenge for Sierra Leone.

According to the WHO's evaluation of Sierra Leone's National Malaria Control Program, Sierra Leone still encounters significant challenges in malaria control. For instance, most laboratories in Sierra Leone have weak testing capacities and inadequate personnel for parasitic diseases and other neglected tropical diseases because of underinvestment. Although technical personnel have been trained in recent years, the overall technical capacity of the country is still inadequate.

The parasitic disease laboratory could be one of the cornerstones for this capacity. Since its establishment in 2017, the parasitic disease laboratory has done field tests for malaria and soil-transmitted helminth diseases as well as vector monitoring. Meanwhile, it is also an important and successful platform in Sierra Leone for training personnel in parasitic disease testing. Laboratory testing capacity of the parasitic diseases should be further enhanced in the future, not only covering malaria control and mosquito vector identification, but expanding to other neglected tropical diseases such as schistosomiasis and filariasis. The laboratory aims to serve as a training and health education base and parasite resource bank in support of parasitic disease control and research in Sierra Leone.

To implement the commitment of China-Africa health cooperation, more effort must be made to explore a model of establishing parasitic disease testing laboratories in countries with high parasitic disease burdens based on public health foreign-aid teams or hospitals where Chinese medical teams work. With the help of the epidemiological knowledge and field work experience from public health foreign aid teams and the clinical resources from medical teams, the Sierra Leone-China laboratory for parasitic disease testing and surveillance could work on multicenter clinical trials for parasite testing and antimalarial drugs and vaccines, which provide technical support for the China-Africa cooperation project on malaria and schistosomiasis control and help spread and share China's experience and products in parasitic disease control and prevention.

Acknowledgments: Ministry of Commerce and the
National Health Commission of the People's Republic of China; Ministry of Health and Sanitation of the Republic of Sierra Leone; directors and all staff of the Center for Global Public Health of Chinese Center for Disease Control and Prevention; all the experts of Phase 2 of the Sierra Leone-China Friendship Fixed Biological Safety Laboratory (Level 3) Technical Cooperation Project (July 2017-June 2020); and Dr. Qin Chen.

Conflicts of interest: The authors declared no competing interests.

doi: $10.46234 / \mathrm{ccdcw} 2021.088$

\# Corresponding author: Ning Xiao, xiaoning@nipd.chinacdc.cn.

\begin{abstract}
${ }^{1}$ National Institute of Parasitic Diseases, Chinese Center for Disease Control and Prevention; Chinese Center for Tropical Diseases Research; WHO Collaborating Centre for Tropical Diseases; National Center for International Research on Tropical Diseases, Ministry of Science and Technology; Key Laboratory of Parasite and Vector Biology, National Health Commission of China, Shanghai, China; ${ }^{2}$ Center for Global Public Health, Chinese Center for Disease Control and Prevention, Beijing, China; ${ }^{3}$ Department of Laboratory Medicine, Central South University, Changsha, Hunan, China.

\& Joint first authors.
\end{abstract}

Submitted: March 01, 2021; Accepted: April 02, 2021

\section{REFERENCES}

1. The World Bank. Sierra Leone. https://data.worldbank.org/country/ sierra-leone?view=chart. [2020-12-28].

2. United Nations Conference on Trade and Development. UN list of least developed countries. https://unctad.org/topic/vulnerable-economies/ least-developed-countries/list.[2020-12-28].

3. World Health Organization. Sierra Leone. https://www.afro.who.int/ countries/sierra-leone. [2020-12-28].

4. World Health Organization. WHO Ebola situation report. https://apps. who.int/ebola/current-situation/ebola-situation-report-30-march2016.[2020-12-28].

5. Tang K, Li ZH, Li WK, Chen L. China's silk road and global health. Lancet 2017;390(10112):2595-601. http://dx.doi.org/10.1016/ S0140-6736(17)32898-2.

6. Hodges M, Dada N, Wamsley A, Paye J, Nyorkor E, et al. Improved mapping strategy to better inform policy on the control of schistosomiasis and soil-transmitted helminthiasis in Sierra Leone. Parasit Vectors 2011;4:97. http://dx.doi.org/10.1186/1756-3305-4-97.

7. GBD 2019 Diseases and Injuries Collaborators. Global burden of 369 diseases and injuries in 204 countries and territories, 1990-2019: a systematic analysis for the Global Burden of Disease Study 2019. Lancet 2020; 396: 1204-22. DOI: 10.1016/s0140-6736(20)30925-9.

8. World Health Organization. Sierra Leone malaria control strategic plan (2016-2020). https://www.afro.who.int/publications/sierra-leonemalaria-control-strategic-plan-2016-2020.[2020-12-28].

9. Dondorp AM, Nosten F, Yi P, Das D, Phyo AP, Tarning J, et al. Artemisinin resistance in Plasmodium falciparum malaria. N Engl J Med 2009;361(5):455 - 67. http://dx.doi.org/10.1056/NEJMoa0808859.

10. Uwimana A, Legrand E, Stokes BH, Ndikumana JLM, Warsame M, Umulisa $\mathrm{N}$, et al. Emergence and clonal expansion of in vitro artemisinin-resistant Plasmodium falciparum kelch13 R561H mutant parasites in Rwanda. Nat Med 2020;26(10):1602 - 8. http://dx.doi.org/ 10.1038/s41591-020-1005-2. 